\title{
ÉLÉMENTS D'UNE CATAPULTE ROMAINE TROUVÉE À LYON
}

\author{
par Dietwulf BAATZ et Michel FEUGËRE
}

Le Musée de la Civilisation Gallo-romaine de I.yon possède dans ses réserves une pièce de grand intérêt, qui fait partie du lot d'antiquités lyonnaises dévolu au musée lors de sa création en 1974, et qui était conservée auparavant dans les collections du Musée des Beaux-Arts. Il s'agil d'une découverte effectuéc. selon A. Comarmond, "en creusant les fondations d'un mur des Antiquailles "1, et dont la nalure n'avait pu ètre identifiée jusqu'à présent. Dans la description qu'il en donnait en 1857. le $D^{r}$ Comarmond notait dejà que le fabricant de cet objet avait fait preuve d'une volonté de solidité évidente. mais, sans grand enthousiasme et à la suite d'Artaud, il émettait l'hypothese d'une "machine hydraulique » (fig. 1 et 2 ).

Grâce à une série de recherches récentes sur l'artillerie romaine, il est possible aujourd'hui d'identifier cet objel comme un élément de atapulte, et de le comparer aux autres machines a trait de ce type qui nous sont connues. Ces déouvertes nous permeltent également de décrire avec précision le fonctionnement de la catapulte, et d'en proposer une reconstitution.

Si l'emplacement exarl de la lrouvaille

1 A. Comannovin, Descriplion des . Inliquilís ol Objels ditrt comlenus dans les sialles du P'alais des. Beaux-.trts de la rille de l.yon. L.yon, 1855-185\%. p. $422, n^{0:} 37-39$. reste mal localisé, on peut néanmoins situer le quartier de l'Intiquaille dans la topographie lyonnaise antique, au cour de l'agglomération qui occupe loute la colline de Fourvière durant, le Haut-Empire. Située au sud du forum, voisine du théâtre et de l'odéon, l'Antiquaille n'est qu'à environ $400 \mathrm{~m}$ de l'emplacement. vraisemblable de la caserne, où stationnaient des l'époque d'Auguste les troupes chargées de la garde de la ville 2 . Il est pourtant difficile de mettre la calapulte de Iyon en rapport avec une enceinte, ou, à plus forte raison, un camp, comme on a pu le faire pour des découvertes analogues du limes ; à Lyon, le problème des fortifications est de ceux qui n'ont pas encore reçu de réponse totalement satisfaisante. encore que quelques découvertes récentes tendent a faire passer dans ce quartier de l'Antiquaille la premiere enceinte coloniale de Lugdunum, puis la muraille du Iaut-Empire (augustéenne? qui l'aurail remplacee ${ }^{3}$.

2 P. Fası, La !rurnison romaine de Lyon, 1918, p. 73-76; A. Armox, Essai sur la Iopographie de Lugdunum, 3e id., Lyon, 1964, p. 111 ; ibid., p. 109 et s., cet auteur évorgure les fouilles el découvertes fortuiles effectures dans l'Antiquialle en 1758, 1826 et 18.45 .

3 A. Arpr, Fonilles Monlíe suint-Barthélemy, dans Lalomus, 1963, p. 733-7.46, al du même, Le mur denceinte ae Lugdumum, dans liull. des Massées el Monuments L.ymmais, IV-1, 1969, p. 171-180, 6 figr., pour les vestiges decourerts en 1962 Montere SaintBarthelemy " enceinte coloniale"; le second article 


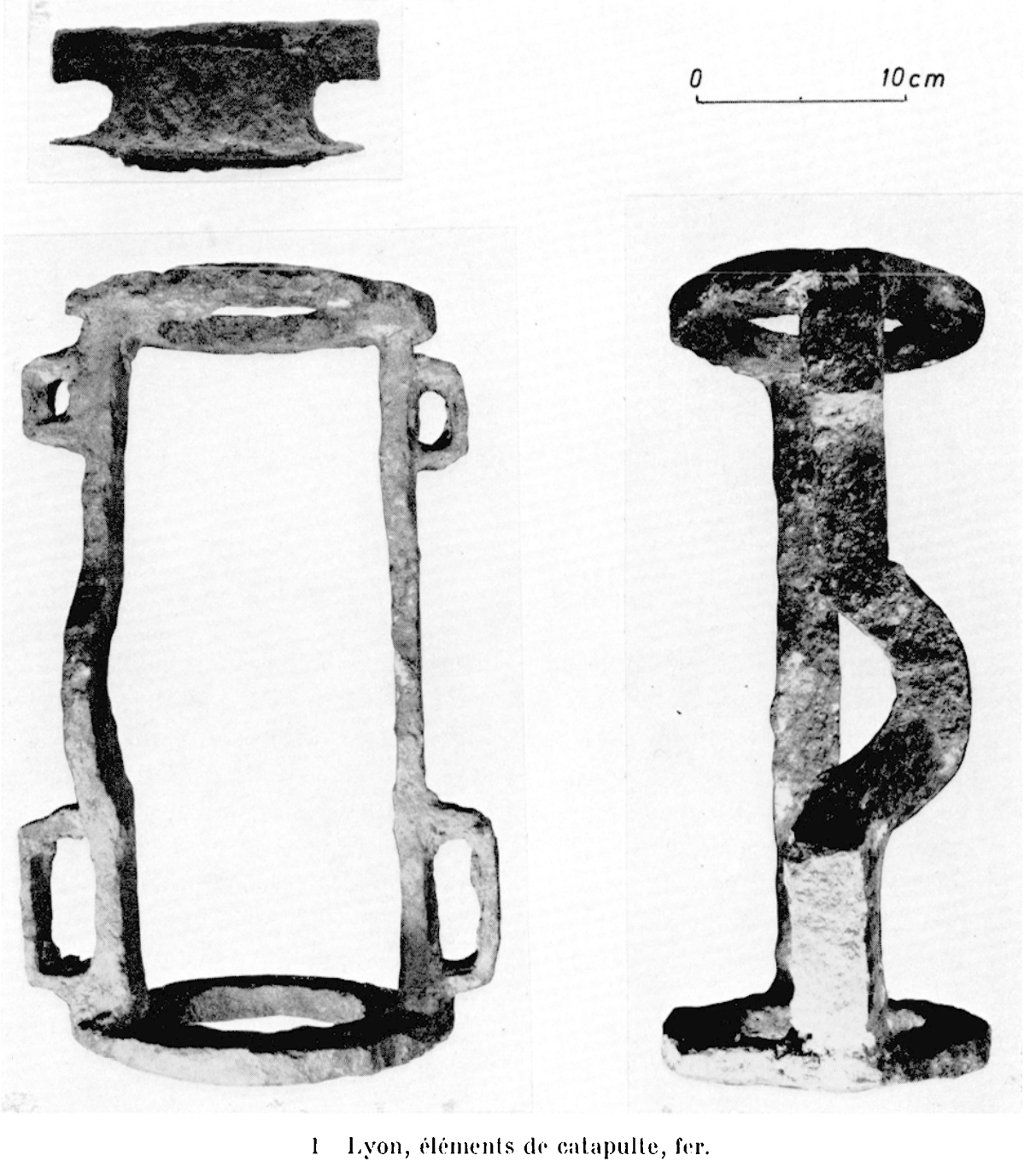

Dans son calalogue du musée, A. Comarmond décrit l'objet de la manière suivante : " $n^{0}: 37$ : Débris volumineux de machine de fer, composé de deux cercles tress épais, grossierement forcés el unis l'un à l'autre, a une distance de $29, \overline{5} \mathrm{~cm}$, par deux bandes de fer ; l'une d'elles est uniforme el a deux bélieres carrées en dehors, dont l'une est plus petite que l'autre; la seconde a également deux bélipres placées de mème, mais elle présente un arceau dans

décril en oul re les deconvertes de 1957. place . Mhelarue, qui concernent une secomble reneeiule, plus vasle.

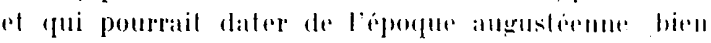
que la mumille ail de suive a cel endroit sur environ $40 \mathrm{~m}$, une seule tour a ele relromian. le milieu (...) ; long. $32,5 \mathrm{~cm} ; \circlearrowleft 22 \mathrm{~cm}$; poids $4,8,() \mathrm{kg} . ")$

"Les numéros 38 et 39 décrivent les parties lalerales, fixees aux deux rercles par trois (sic) rivels de fer ; largeur $15,8 \mathrm{~cm} ; \mathrm{ht} .8 \mathrm{~cm}$; poids 1,34:3 al 1,410 ku. "

Depuis quelques annees, on connait une découverte lout à fail identique faite à Orşova en Roumanie (fig. :3 et 4). Eille fut trouvée en mème lemps qu'une lige de fer possédant des extrémités fourchues el montrant en son rentre un arreatu. Ces deux objets furent, derouverts par X. Gudea au cours de la fouille d'un petil retranchement d'époque romaine 


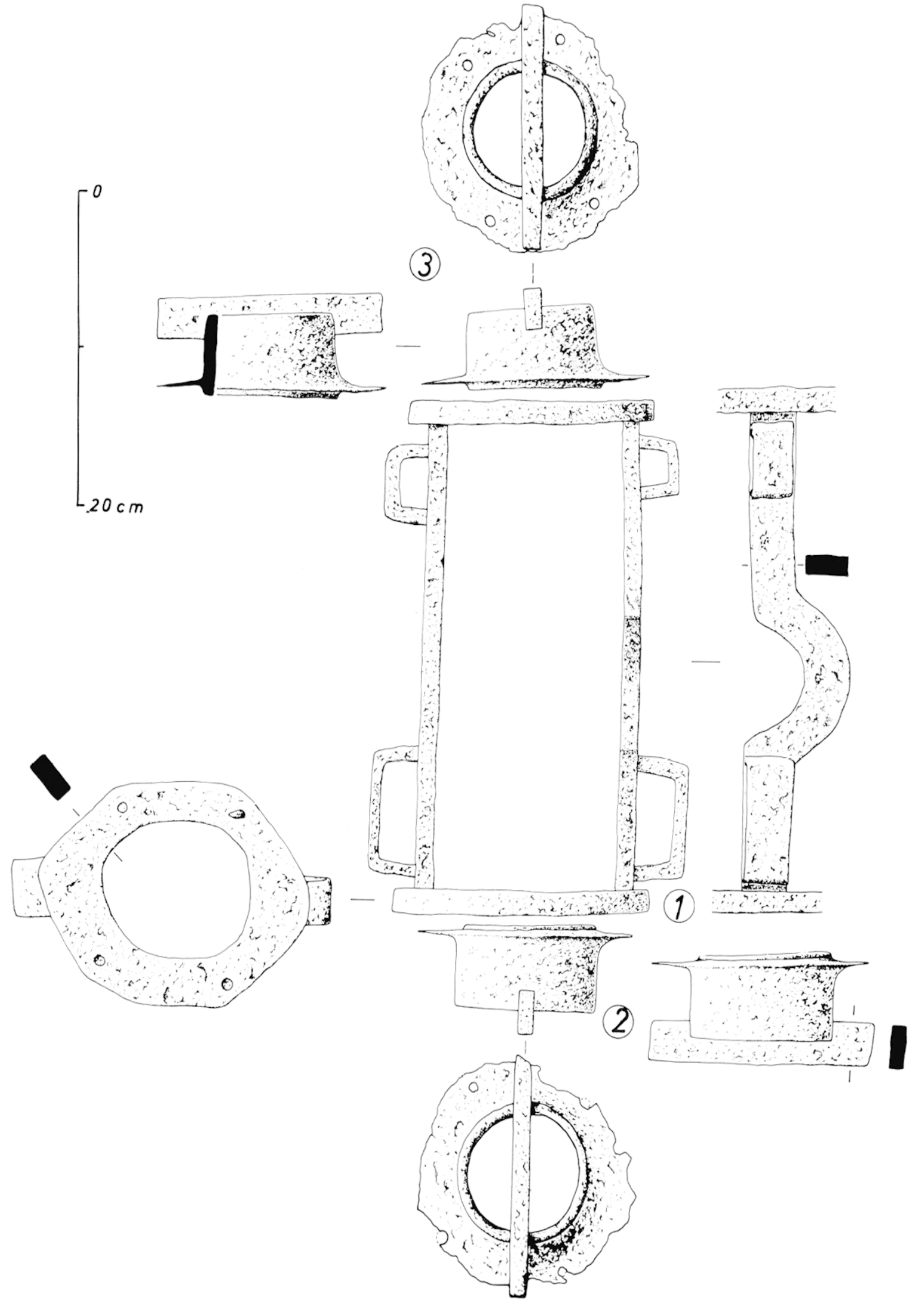

2 lyon, doments dr calapulte. 1. Kambestrion; :-3, modioli. 


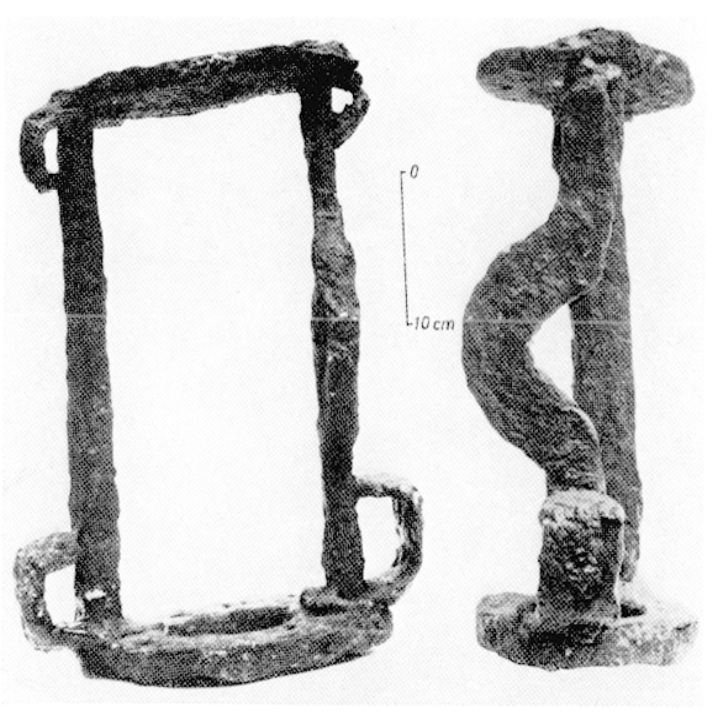

3 Orşova, kambestrion d'une catapulte, fer.

4 Orşova, kambestrion d'une catapulle.

5 Orsova, lamarion diune catapulte. $\rightarrow$
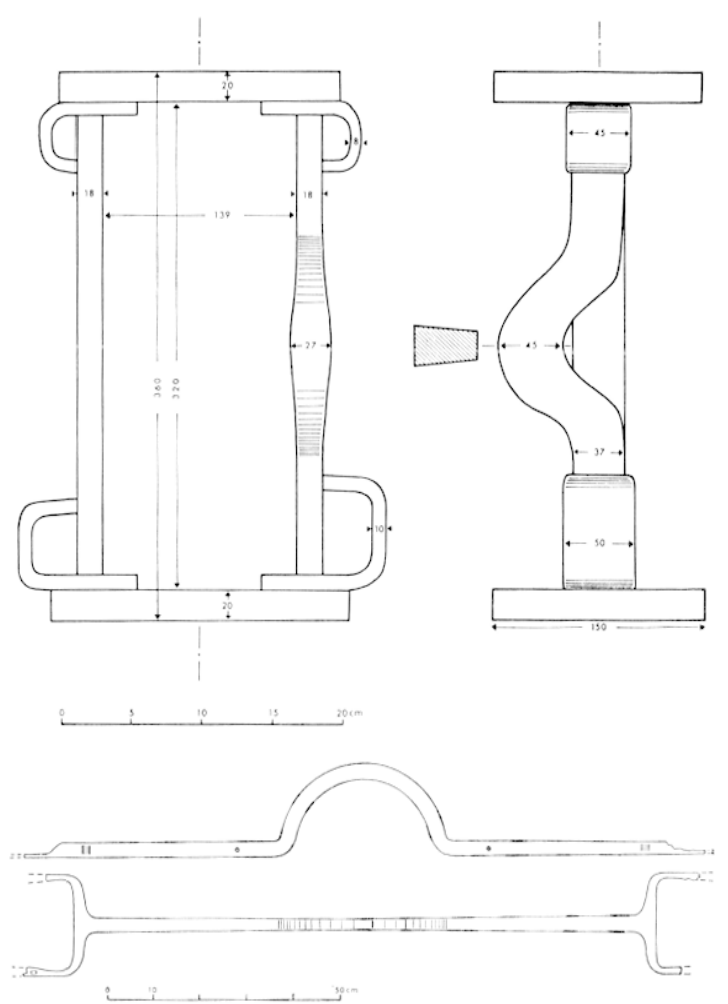

pu ètre interprété clairement que depuis quelques années, grâce aux travaux de E. W. Marsden? ${ }^{7}$. Celui-ci a pu montrer que le texte décrivait un système de torsion à deux bras, construit de la même manière que la catapulte de la colonne trajane (fig. 6) ${ }^{8}$. Les illustrations antiques de la Cheiroballistra, qui, au Moyen Age, ont été recopiées en même temps que le texte et nous sont ainsi parvenues, sont d'une aide précieuse pour cette interprétalion (fig. 7$)^{9}$. Il s'agit de dessins techniques illustrant les différentes parties de la catapulte décrite dans le texte de la Cheiroballislra.

De l'étude du lexte et des illustrations de la Cheiroballislra de Héron, il résulte avec

7 L. W. Marsuen, Greel and Roman Artillery, 1, IIistorical Development, Oxford, I969, p. $189 \mathrm{ct}$ s.; II, Technical Treatises, Oxford, 1971, p. 206-233.

$\checkmark$ E. W. Marsnen II, loc. cit., p. 208-209.

9 Marsden n'a pas pris en consideration les illustralions anliques du lexte; elles sont reproduites par sehneider (op. cil.) el l'avaient ele anparavant dans la publication de la Cheiroballistra de Héron par Wescher : C. WEscmen, Poliorcálique des cirecs, Jaris, 1867 , p. 121-134. 


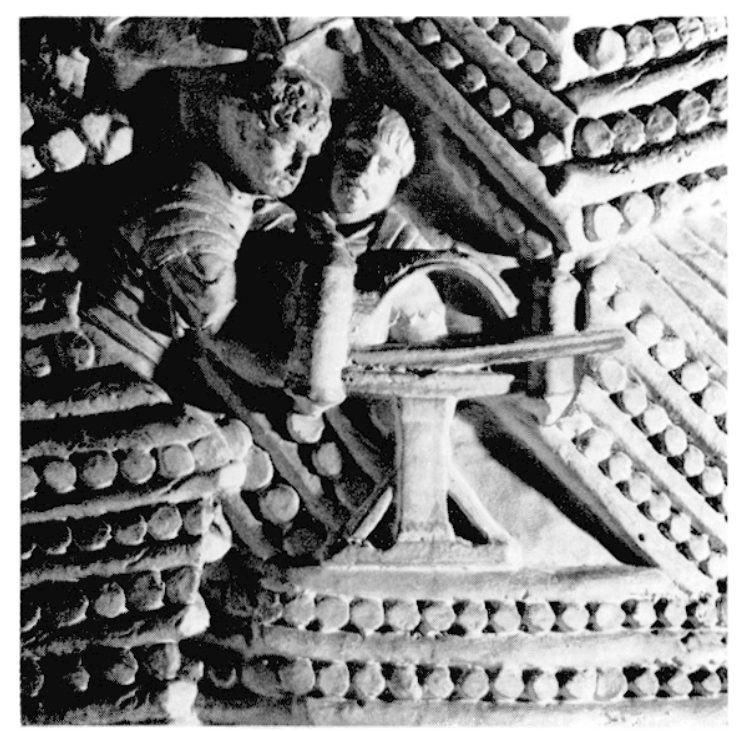

6 Rome, colonne trajane, catapulle en position de tir: les hambestria arec leurs faiscallx sont proleyes par des manchons de tole.

certilude que l'on a découvert à Orşova un kambestrion (cadre de tension; fig. :) et 4) et un kamarion (arc-boutant; fig. 5). Comme. d'après la description. il doit y avoir deux kambestria, el encore d'autres éléments métalliques, la découverte d'orşova ne comprend donc pas toules les parties mélalliques d'une catapulte.

La découverte lyonnaise ne comprend ainsi qu'un lambeslrion (lig. 1 et 2, 1); elle est donc. elle aussi incomplete. Mais avec le fambestrion ont été conservés les barillels qui s'y adaptaient, munis de leurs clavettes (ficr. 2. 3-4), alors que ces éléments manquaient à Orşova. A Lyon, ils sont en fer, et celle déouverte contribue heureusement a approfondir nos connaissances. Chaque barillet romporte une clavette en fer placie en travers de louverture.

D'apres la description de la Cheiroballistra. les deux lambestria d'une catapulte comprenaient quatre barillets munis de leurs quatre claveltes : ces différents éléments sont figurés sur les illustrations antiques de la Cheiroballistra : fig. 7, 1, a droite. on reconnait en bas les 4 barillels el. all-dessus. les 1 clavelles qui doivent se placer dans lemenehe ménatere dans la partie superieure du barillel.

Vitruse designe ce barillet sous le nom
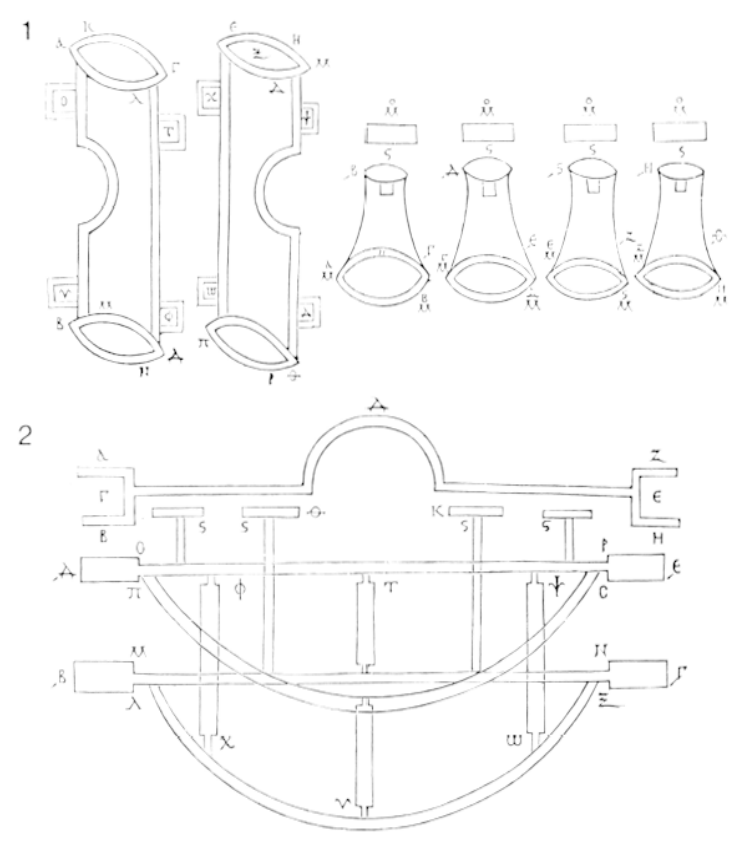

7 Copies modievales des illustrations antiques du lexle de Iferon, la cheiroballistra dapres C:. Wescher ; 1, à gauche; deux kambestria: 1, a droile; qualre modioli ave leurs clavelles (epizygldes); 2, en haut: arc-boutanl (kamarion) ; 2 , en bas : liranl (ladmalion).

de modiolus ${ }^{10}$. De son lemps, sur de petites catapultes, celle partie était pratiquement toujours en bron\%e (fig. 8). Les deux modioli de Iyon sont les premiers de ce lype que l'on connaisse, en fer ${ }^{11}$. Vitruve mentionne également la clavette sous le nom d'epizygis ${ }^{12}$; celle-ci est toujours en ler.

Fonction de la calapulle. Les fragments de Iyon appartiennent a une pelite machine a trait, à deux bras, fonctionnant par lorsion el destince a tirer des fleches. Les systemes de torsion a deux bras ont été vraisemblablement mis au point au miliru du re s. ar. J.-C.

11) Vitrute, $x, 11,5 ; 1: 1$.

11 Des modioli de bronze sont commus sur les sites suivants : Ampurias Espagno, voir fig. 10; Ephyra (Grice: : I). BAAт\%, Archäol. Anzeiger, 1979, p. 68-75; Cremone Ilalio: (i. Poxrarol, Cal. della Sezione Archeologica del Musen Givica ". Ala Ponzone" di

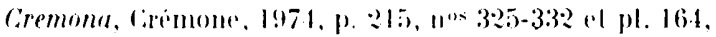

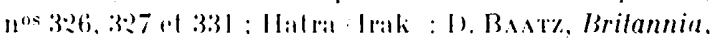

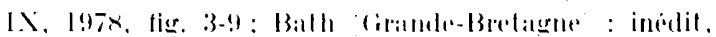
aimable renseignement are 13. Cimbliffe.

$1:$ Vitrive, $x, 11,1 ; 1: 1$. 


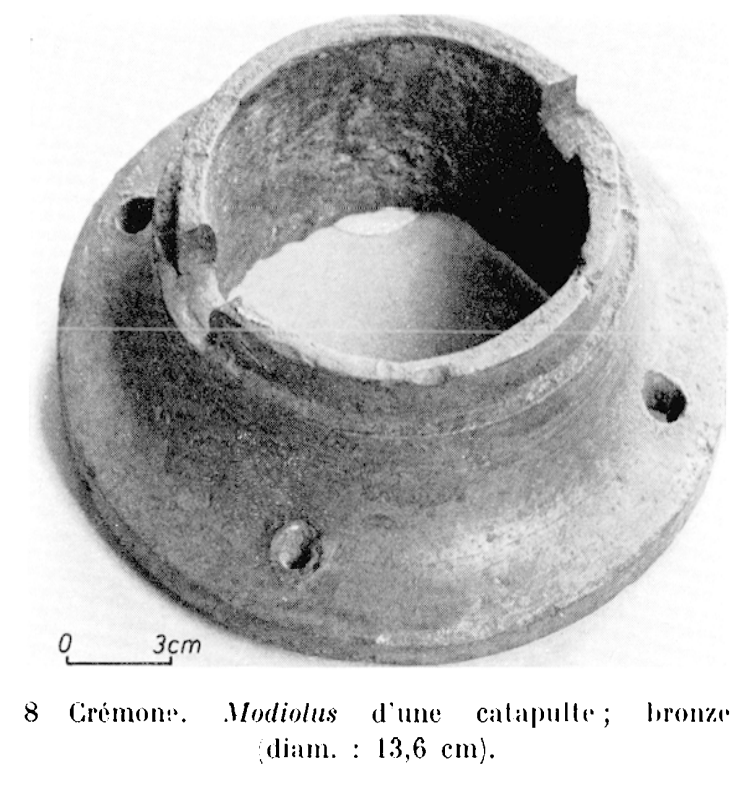

par des lechniriens oreces ${ }^{13}$. I l'epoque hellenistique, ces calapultes a torsion ont été ameliorées ef standardisés: elles nous sont essentiellement connues par les descriptions délaillées de Héron. Philon el Vitruve. Ies radres de lorsion des ralapultes hellénistiques étaient en bois. Sourent, ils comprenaient des placages mólalliques resistants, a peu pris romme la ralapulte d'Ampurias tig. 9/14: celle-ci appartient à la premiere moitié du $\mathrm{II}^{\mathrm{e}}$ s. av. J.-C. La reconstitution de la calapulte d'Ampurias montre Iris distimctement les deux faisceux de torsion (fig. 10).

Un faisceau de torsion était conslitué d'une corde embobinée, dont la fibre elait faite de crins ou de lendons animaux. Iux deux extrémités. la corde passail chaque fois a travers l'un des barillets en rontournant la clavelle en fer placee en lavers de l'ouverture (fig. 11). lors de la fabrication de la calapulte. on plaçait déja la corde en lui faisant subir une forte lension ${ }^{15}$. Les bras de la calapulte, en bois, étaient insérés en travers du faisceau. On les

13 F. W. IARSIL:N 1, loc. cit., P. 16; Y. (BARIAX, Recherches de poliorcílique grecque, Paris, 1974, P. 166$16 \times$ at $213-2 \cdot 25$.

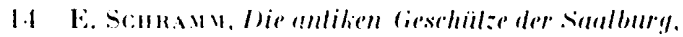
Berlin 1918. p. 11-16 (21 75-77.

15 Vitrivi, $X, 12,1-2$.

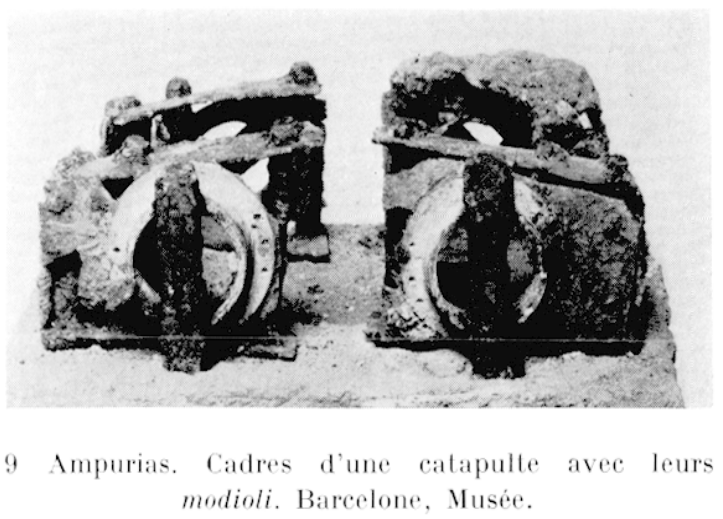

fixail à une corde tine mais solide jouant le mème ròle que la corde d'un arc. La corde étail retenue en son centre par un système de rage qu'il fallait, pour tendre la catapulte, lirer en arriere a l'aide d'un treuil. Quand on armait. les bras de la calapulte pivotaient en tordant les faiscealux de libres pré-tendus : c'est de lia que vient l'appellation de système a torsion (tormenlum). Lal construction ingénieuse du faisceau de torsion avec barillet (modiolus) et clavelte (epizygis) permet de doser exactement la tension de la bobine (et ce à l'aide d'une grande clé en forme de levier), done de doser la puissance selon la nécessité. Cetle possibilité de réglage est d'une grande importance pour assurer une utilisation efficace de la calapulte. C'est pourquoi on retrouve les modioli dans toute catapulte à torsion. Lne fois la pré-tension effectuée, les modioli étaient fixés et assurés sur le cadre par des fiches (fig. 11). De cette façon, pendant le tir, il leur étail impossible de revenir en arrière par suite des vibrations. Les trous pour ces fiches existent aussi dans la trouvaille de Lyon, aussi bien dans les modioli que sur le liambestrion. Chaque anneau du kambestrion comporte + trous: a cause de lia corrosion avancée, le nombre des trous des modioli est difficilement visible.

1 la fin du $1^{\text {er }}$ s. alp. J.-C.. les Romains mirent au point un nouveau modile de calapulte à cadre entiorement métallique. Il devail remplacer, en ce qui concerne l'artillerie légerer. les anciens exemplaires hellénistiques is cardere en bois. La nouvelle arme fut utilisée pour la premiere lois par Trajan au cours des 


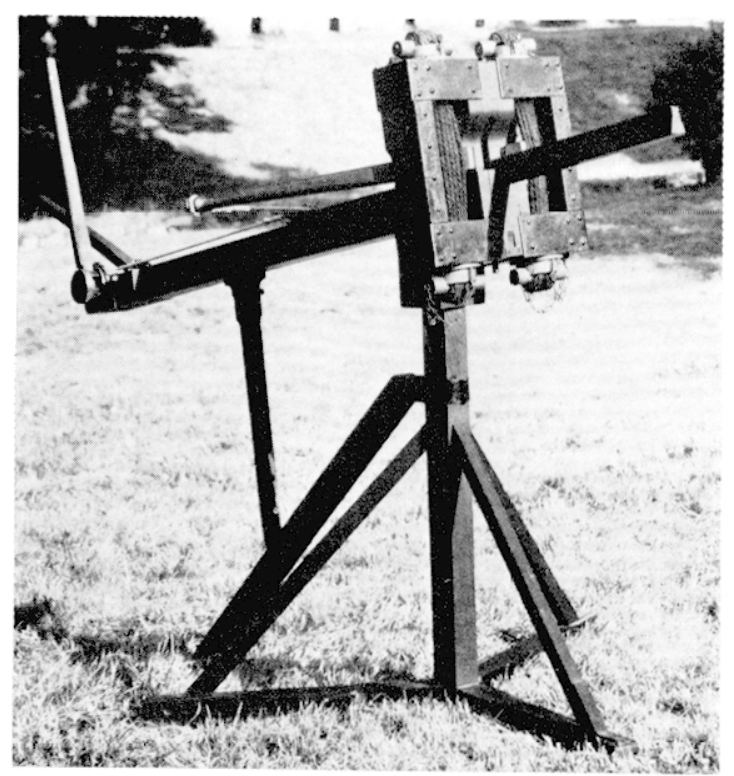

10 Reconstitution de lat calapulte d'Ampurias. Bad-Hamburge, Saalburerr-muse'um.

guerres daciques, el à ce lilre, elle est figurée sur la colonne trajane (fig. 6). Ce lype de catapulte a ensuile été utilisé pendant plusieurs siecles sous de multiples variantes, et cela jusqu’à l'époque byzanline. I al seule description suftisamment détaillée qui nous ait élé conservée, d'une variante de la calapulte à cadre métallique, est le lexte de la Cheiroballistra mentionné ci-dessus, el qui date de l'époque romaine tardice ou byzantine' ${ }^{16}$. C'est à ce modele à cadre mélallique qu'appartient également la découverte lyonnaise.

Le lambestrion et les deux modioli de Lyon formaient le cadre de l'un des deux faisceaux de torsion. Avec l'enroulement de crins ou de tendons, on obtenail un faisceau complet qui pouvait se fixer facilement sur le cadre métallique de la calapulte : c'élait un module amovible. I'essai de reconstitution (fig. 12) donne une idée de l'aspect que pouvail prendre la catapulte de Lyon, avec le mode de fixation du lambestrion le plus vaisemblable. Celte

16 E. W. Marsinex I, loc, cil, p. 2009, place le

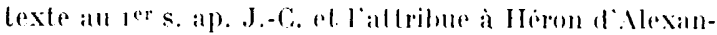

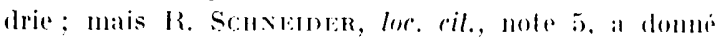
des arguments detepminamls pour ume dalalion latrdive. prouvant done que ce lexte me pouvat elre attribur a Iféron d'Mlexandre.

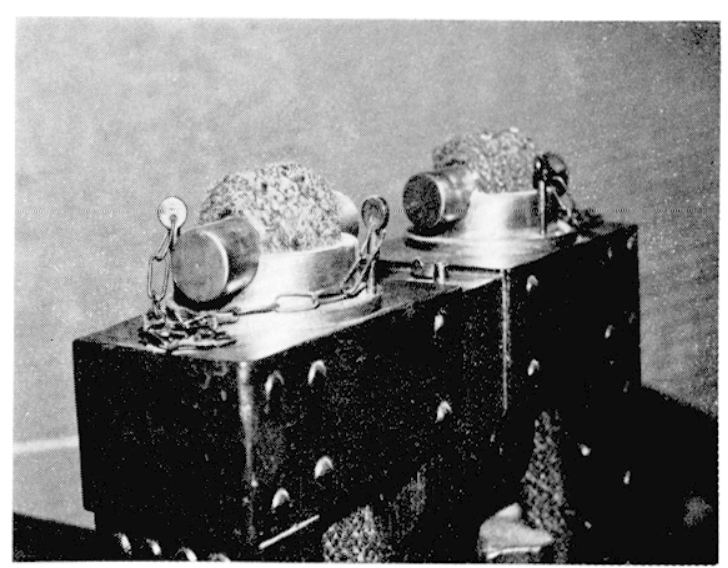

ll Reconslitulion de la calapulte d'Ampurias. Detail des modioli.

reconstilution a pul ère realise grace aux découvertes d'Orşova el de Ixyon, au lexte de la Cheiroballistra el a quelques éléments de la description d'une catapulte par Vitruve ${ }^{17}$. Cne housse de protection cylindrique, en tòle. était encore placée sur le liambestrion pour le protéger du mauvais temps et des armes ennemies (lig. 6).

Taille et proportions de la calapulte. Etant donné que ces éléments de catapulte sont en fer, et fortement corrodés, il n'est pas possible d'en prendre des mesures lris exactes. On peut néanmoins donner quelques indications sur la taille et les proportions de l'arme.

Dans les calapultes hellénistiques standardisées qui sont décrites che\% IÉron. Philon et Vitruve, les dimensions de chaque piece importante correspondent à des multiples ou a des fractions d'une mesure de base. Celle-ci est donnée par le diamètre des ouvertures circulaires pratiqués dans le cadre en bois pour le passage des faisceaus de lorsion. Ce diamèlre est idenlique au diamolre interne du barillet (modiolus). Vitruve désigne la mesure de base sous le nom de foramen.

Il a dù vraisemblablement exister des descriptions techniques du mème genre pour

17 Le texte de la Cheiroballistra decerit une petile arme a forsion, qui pouvait èlre manreurese a la main

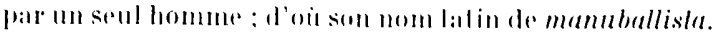

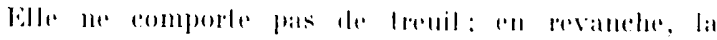
calapulte de lyon dat plus gramde que la cheiroballistra : elle necessibail done lousage d'un lonil. 


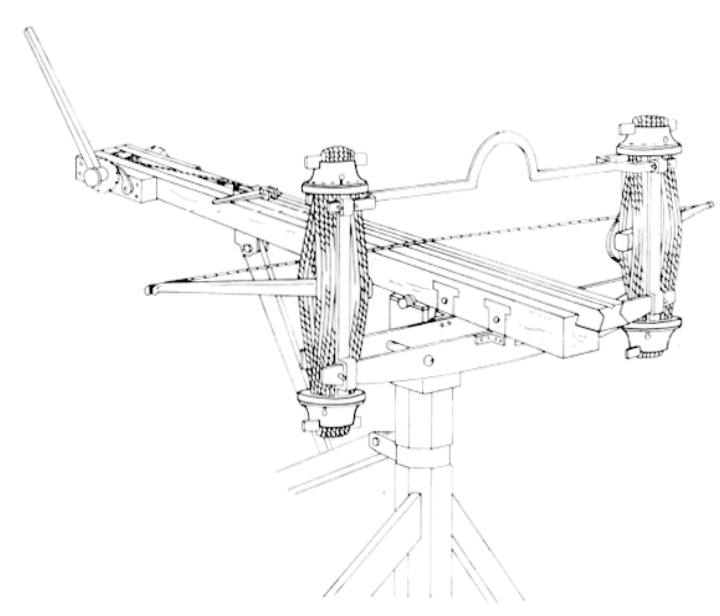

12 Essai de reconstitulion de la catapulte de Lyon. l.es manchons do tòle protégeant les lambestria ne sont pas figures.

les pièces d'artillerie plus tardives, à cadre métallique, du type de la catapulte de Lyon; et dans ces descriptions, toutes les dimensions devaient découler elles aussi d'une mesure de base; cependant, aucune de ces descriptions ne nous est parvenue. Le texte de la Cheiroballistra décrit bien une arme de petite taille, à cadre métallique, mais il ne fait pas dériver ses dimensions d'une mesure de base.

Pour la calapulte de Lyon. comme pour les anciennes armes hellénistiques, c'est sans doute le diametre intérieur du modiolus qui a servi de mesure de base; il s'élève à $7,5 \mathrm{~cm}$ environ, c'est-à-dire à peu près 4 digiti ${ }^{18}$. Cette mesure de base a dû être fréquemment employée. On la rencontre également sur la catapulte plus petite de Crémone, mais en fait celle arme du I $^{\mathrm{er}}$ s. ap. J.-C. appartient encore au vieux type hellénistique à cadre en bois ${ }^{19}$. La catapulte hellénistique des cités et des états grecs utilisait principalemenl une mesure de base égalanl 4 daclyloi. D'apros les règles de construction des auteurs anciens, elle envoyait des flèches d'une longueur 9 fois supérieure, soit $: 36$ daclyloi $=: 3$ spilhame. el

18 I digitus $1, x=$ cm, soil la seizieme partir du pied romain a de $29,6 \mathrm{~cm}$ !

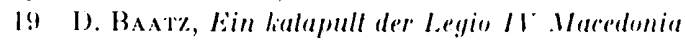
aus Cremona, dans Romische Milleilungen, $\times 7,19 \times 0$, 1). $2 \times 3-2999$.

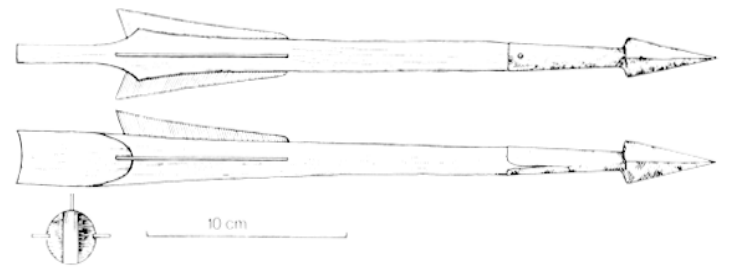

13 Trails dr calapulle romaino plus tardive, espuisse d'apres les decouvertes de Dura-Furopos (milieu du 1$]^{\circ}$ s. ap). J.-(.. Le. Les trois ailerons de stabilisation sont en lamelles de hois mince.

c'est pourquoi elle étail connue sous le nom de "catapulte trispithamique ${ }^{20}$.

C'est apparemment au ier s. ap. J.-C., lors du passage des catapultes de type hellénistique (à cadre en bois) aux armes à torsion (à cadre métallique), qu'on a dû adopter de nouveaux types de traits. C'est la raison pour laquelle on peut admettre que la catapulte de Lyon ne langait pas des flèches effilées (ayant 9 fois la longueur de base, soit $67, \overline{\mathrm{cm}}$ ) comme le prescrit Vitruve, mais plutòt des traits courts, plus robustes, du type de ceux qu'on a découverts à Dura-Europos. Ceux-ci ne mesuraient que la moitié de la longueur des flèches utilisées sur les anciennes catapultes, et avaient un poids d'environ 60 à $100 \mathrm{~g}$ (fig. 13) ${ }^{21}$. La portée maximale des calapultes légères à flèche atteignait environ 300 à $600 \mathrm{~m}$, la précision étant optimale entre 50 et $150 \mathrm{~m}^{22}$.

20 Pour la longueur des flèches utilisées par les plus anciennes machines à trait : PHilox (éd. Marsden II, loc. cil.) 54, $28-55,1$; Vitruve X, 10, 1. En ce qui concerne la fréquence de la catapulte trispithamique: E. Scinамm, loc. cil., p. 22. E.-W. Marsinen, I, loc. cil., p. 70, 171. I.es catapulles de celle taille sont igalemenl friefuentes d'apres les decouvertes.

21 F. Cinoxt, Fouilles de Dura-Europos, Paris, 1926, p. 260, pl. 97, 1-2; The Excavations at DuraEuropos; Preliminary Reporl of Second Season of Wort, Now llaven 1931, p. 7\%, pl. 9; Preliminary Reporl of Sirlh Season of Work, New Haven, 1936, p. 455, fig. 24, 2. Ine bonne description d'une fleche de ce lype est donnée par Procople, Bell. Golh. 1, 21, $15-16$.

22 Arec la reconslitulion de la calapulte d'Ampurias, Schramm a alteint ume porté maximale de 305 m (loce cil., p. 7ti . I a portio la plus lomgure que l'on puisse admettre pour more ralapulte legreme antigue nous est rapporter par Athenaios Ilech. fod. C. Wescher $8,5-9$, aree 3 1/2 slades soit un peu plus de $600 \mathrm{~m}$; 
Si nous désignons par f la mesure de base (foramen) utilisée pour la calapulte de Lyon (f égalant env. $7, ; \mathrm{cm}$ ), le diametre extérieur du modiolus atteint pratiquement $2 f$ et sa hauteur, environ $3 / 4 f$. Ce sont justement les proportions prescrites par Philon et Vilruve pour les anciennes armes à torsion ${ }^{23}$. Les clavettes (epizygis) de Lyon ont une longueur de $2 f$, et leur section de $1,5 \times 3 \mathrm{~cm}$ correspond de manière extraordinaire à celle que transmet Philon, qui est de $1 / 5 f \times 2 / 5 f^{24}$. Les barillels et claveltes de la catapulte lyonnaise suivent donc encore la tradition hellénistique. Naturellement, ces proportions sont aussi déterminées par des impératifs techniques.

La distance entre les deux anneaux du kambestrion est très exactement de $4 f$; c'est aussi le cas pour le kambestrion d'Orşova. qui est cependant un peu plus grand puisqu'il a été conçu à partir d'une mesure de base de $7,9 \mathrm{~cm}$. Les autres mesures du kambestrion consistent également en multiples ou en fractions du foramen. En fait, on ne peut faire aucun rapprochement avec les sources concernant l'artillerie à torsion hellénistique, car

là-dessus voir E. W. Marspex, 1, loc. cit., p. 88. A des portées supérieures, la précision du lir diminue : a cause de la résistance de l'air, la vitesse de tir du trait et du mème coup sa force d'impact diminuent conside. rablement. Dans la plupart des cas, le fail de tirer a des distances supéricures aurail donc eté lactiquement insensé.

23 PHulox éd. Marsden II, loc. cil.), 53, 10-13; Vitreve $X, 11,5$.

24 Philon éd. Marsden II, loc. cil. 53, 23-24. ces armes ne possédaient pas encore de liambesIrion mélallique. Toutefois, la présence de rapports de mesure bien déterminés sur certaines parties de la catapulte de Lyon semble indiquer qu'il a existé pour l'artillerie à torsion tardive, à cadre métallique, des règles de construction analogues à celles qui nous sont données, pour les catapultes plus anciennes, par les descriptions de Héron, Philon et Vitruve.

Ainsi, la pièce trouvée à Lyon constitue une partie d'une catapulte légère tirant des flèches. Celte catapulte appartient a un type mis au point par les Romains vers la fin $d u{ }_{1}$ er s. ap. J.-C., et qui a été utilisé pour la première fois dans les guerres de Trajan contre les Daces. I.e type en a été longtemps conservé, ct on le trouve encore à l'époque byzantine. La trouvaille de I yon a dû ètre enfouie postérieurement à 100 ap. J.-C., peut-être lors des combats entre Septime Sévère et Claudius Albinus, à l'occasion desquels la ville de Lyon fut en partie détruite.

\section{BaAtz et II. Feugìre}

N. B. - M. Fengire est l'auteur de la découverte, el la présente dans son contexte lyonnais; D. Baatz s'rst chargé de l'étude proprement dite de la catapulte; les auleurs tiemnent à remercier .11. A. Audin, conservateur du Musée de la Civilisation Gallo-Romaine, qui a bien voulu auloriser celte étude, et dont lo rendre, $\Lambda$. Finas, s'était intéressé à cet objet avant de disparaître prematuriment en 1974. Fr. Leyge, photographe du musée, est l'auteur des clichés de la fìg. 1 , et I). Ferra a facilite les problimes de traduction. 
